\title{
SURGICAL MANAGEMENT OF UNCOMPLICATED HALLUX VARUS DEFORMITY IN AN ADOLESCENT
}

Arnab Karmakar ${ }^{1}$, Tanmay Datta르, Sunil Kumar Das ${ }^{3}$, Ananda Kisore Pal ${ }^{4}$

\section{HOW TO CITE THIS ARTICLE:}

Arnab Karmakar, Tanmay Datta, Sunil Kumar Das, Ananda Kisore Pal. "Surgical Management of Uncomplicated Hallux Varus Deformity in an Adolescent". Journal of Evolution of Medical and Dental Sciences 2014; Vol. 3, Issue 14, April 07; Page: 3652-3656, DOI: 10.14260/jemds/2014/2336

ABSTRACT: A thirteen year male patient attended our Out patients department seeking treatment for deformity of the right great toe which was present since birth. The right great toe was deviated medially and ventrally from the proximal metatarsophalangeal joint. The angulation was even more on the interphalangeal joint. There was an accessory toe on the inner aspect of great toe originating from the level of metatarsophalangeal joint, a thick tight cord was also palpable attached to it and extending upto base of first metatarsal. The radiography reveals the same findings and traces of extra bones on the inner side. We choose the Farmer's technique for repair with few modification and addition. On six months follow up the correction is fully maintained \& the metatarsophalangeal is having ten degrees of both dorsiflexion and plantar flexion.

KEYWORDS: Hallux, Varus, Farmer's technique

INTRODUCTION: Congenital Hallux varus is a comparatively rare deformity, only few cases have been reported in the literature. In this deformity the great toe is angled medially at the metatarsophalangeal joint. It should be differentiated from the varus deformity of the first metatarsal (metatarsus primus varus). ${ }^{1}$ The deformity angle may vary from zero to ninety degrees. The deformity is mostly unilateral, but bilateral cases also have been reported. The condition itself is more unsightly than disabling. The following case is reported because of its rarity \& relatively easy management.

PRESENTATION: A thirteen year male patient attended our Out patients department seeking treatment for deformity of the right great toe which was present since birth. The main complaints made by the patient was that it was very difficult to wear a shoe, he cannot move the toe to its normal position voluntarily, and there was presence of an extra toe on the inner side.

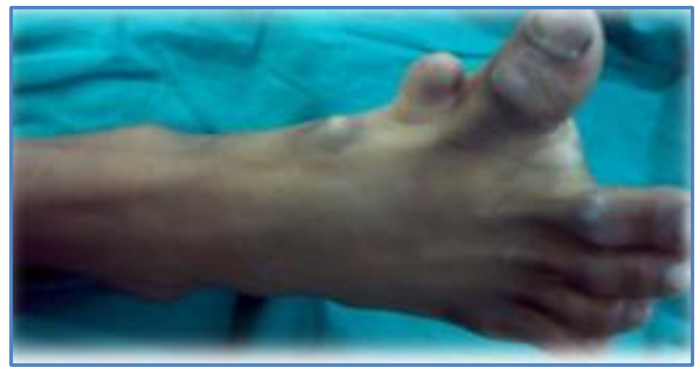

Figure 1a

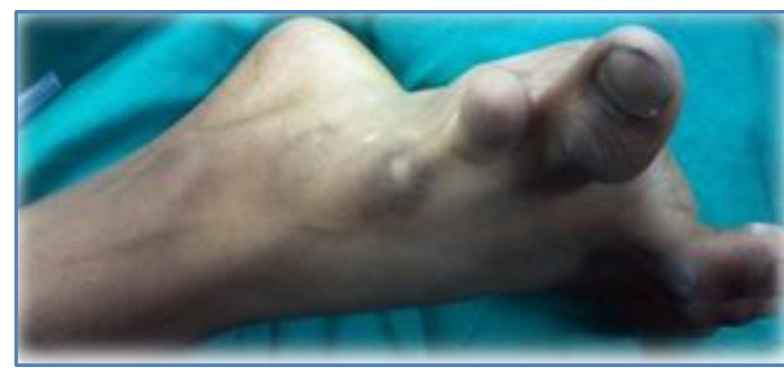

Figure 1b

Fig. 1 a, b; showing the deformity, accessory toe \& the visible band. 
EXAMINATION: The right great toe was deviated medially and ventrally from the proximal metatarsophalangeal joint. The angulation was even more on the interphalangeal joint. There was an accessory toe on the inner aspect of great toe originating from the level of metatarsophalangeal joint, a thick tight cord was also palpable attached to it and extending upto base of first metatarsal. The range of motion of the metarsophalangeal joint was restricted compared to normal side, rest joints showed normal motion. There was $3 \mathrm{~cm}$ gap between great toe and second toe. Rest of the foot reveals no other abnormality. The radiography reveals the same findings and traces of extra bones on the inner side.

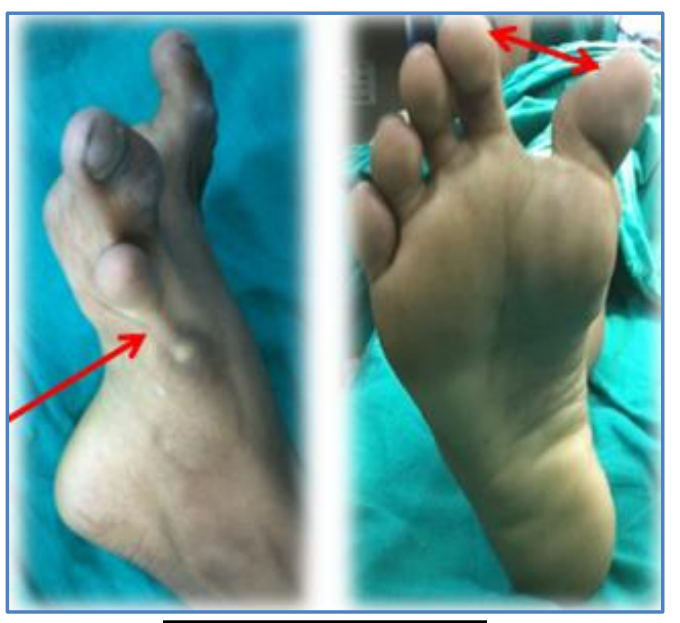

Figure 2a \& 2b

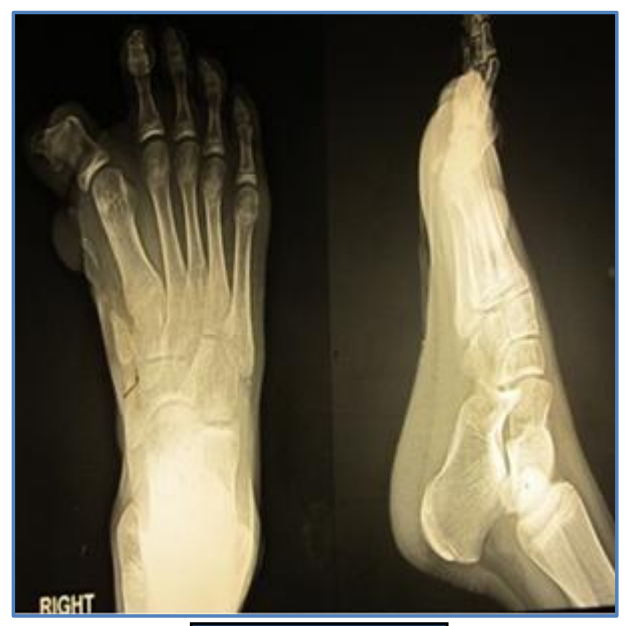

Figure 2c

Fig. 2a, b, c: a) showing clearly the fibrous band, b) showing the gap between great and second toe, c) radiograph showing the deformity and accessory bone.

TREATMENT OFFERED: As the boy had growing bones our aim of treatment was to restore the anatomy as much as possible maintaining the full joint functions. So after considering all the available treatment options we choose the Farmer's technique for repair with few modification and addition. ${ }^{2}$

SURGICAL PROCEDURE: Under spinal anesthesia after application of tourniquet, a broad Y-shaped flap of skin and subcutaneous tissue from the dorsal surface of the web between the first and second toes was raised; keeping base the flap dorsally in the space between the first and second metatarsals, and include in it the skin contiguous with the web distally along the two toes for one third of their length. From the medial edge of the base of the flap, the incision was curved medially and slightly distally across the medial aspect of the first metatarsophalangeal joint. The incision was then dipped transversely through the medial part of the capsule of the first metatarsophalangeal joint.

A syndactyly between great and second toe was made by suturing the opposing skin edges together. The metatarsophalangeal joint was reduced after opening the joint capsule and a smooth longitudinal Kirschner wire was inserted from the tip of the great toe into the first metatarsal to align the great toe in a neutral position. Then the accessory phalanx along with the fibrous band was resected out. The Y-shaped flap of skin and subcutaneous tissue turned medially, and sutured in place to cover the defect in the skin on the dorsal and medial aspects of the first metatarsophalangeal joint. 


\section{CASE REPORT}

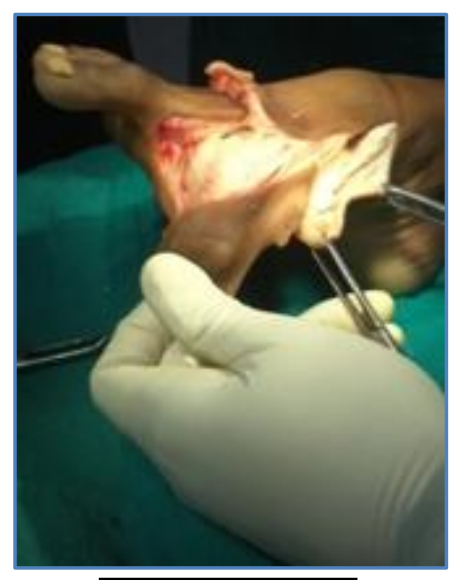

Figure 3a

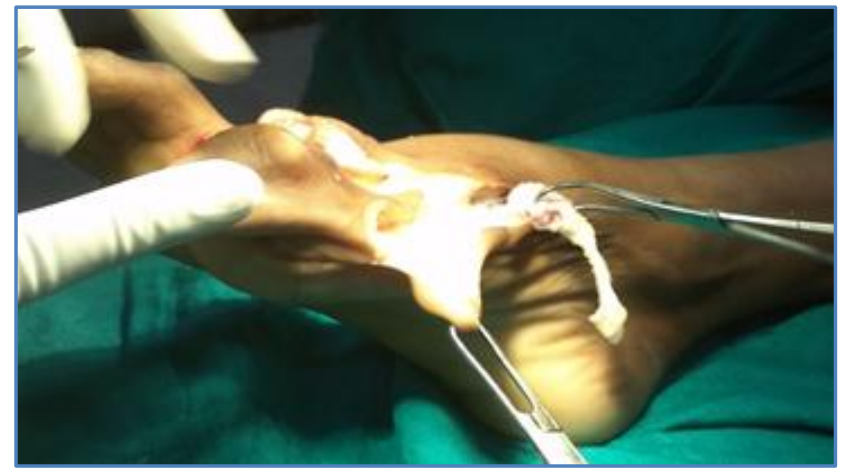

Figure 3b

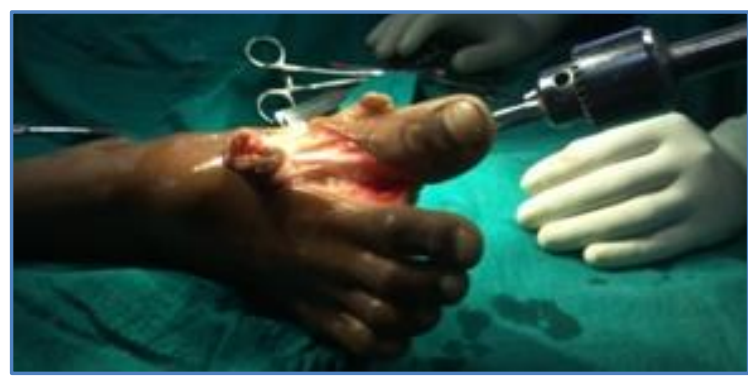

Figure 3c

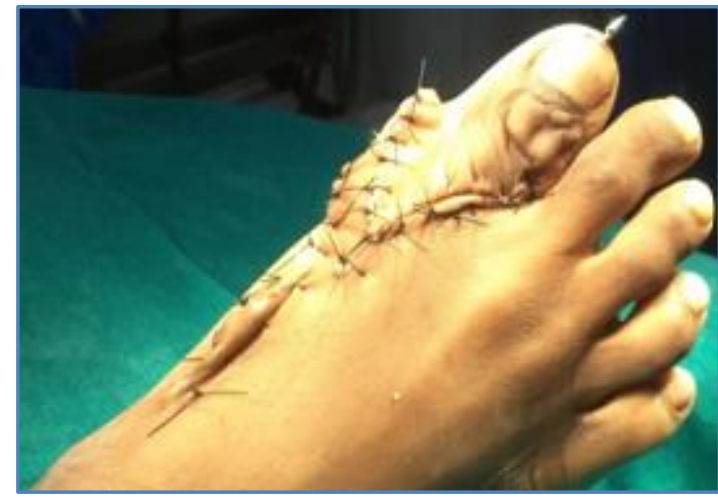

Figure 3e

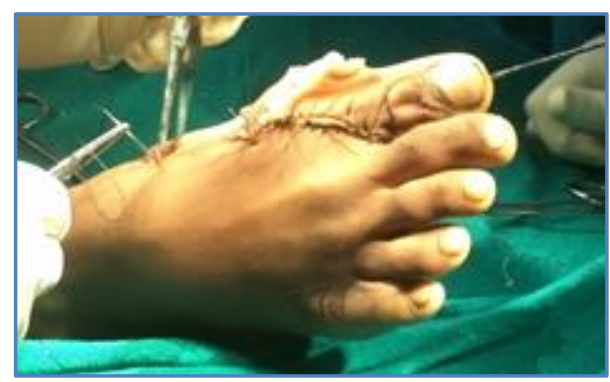

Figure 3d

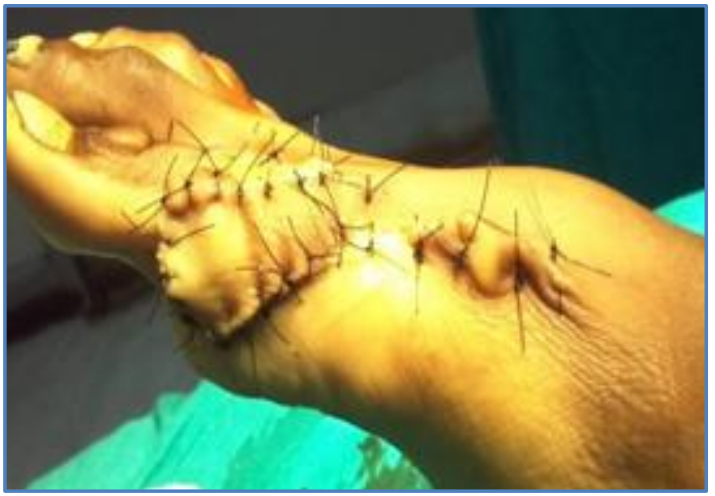

Figure $3 f$

Fig. 3a, b, c, d, e, f: a) the Y shaped flap; b) excising the fibrous band; c) fixing the joints with k-wire; d) doing syndactyly; e, f) after final placement of the flap.

POST-OPERATIVE REHABILITATION AND FOLLOW UP: A below knee slab was given post operatively which was kept for six weeks. Wire was removed after six weeks and active movement started. Post-operative period was uneventful except mild skin slough, which healed after few days of 
dressing. On six months follow up the correction is fully maintained \& the metatarsophalangeal is having ten degrees of both dorsiflexion and plantar-flexion.

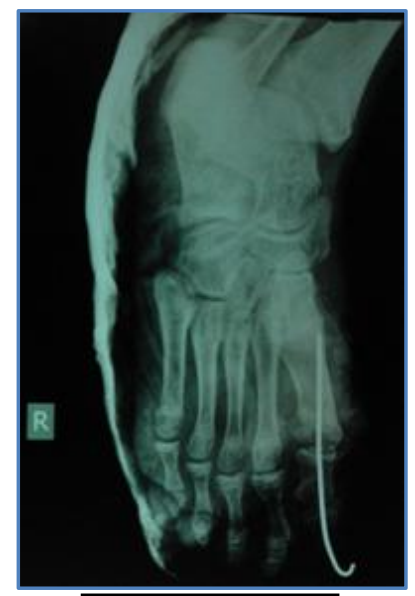

Figure 4a

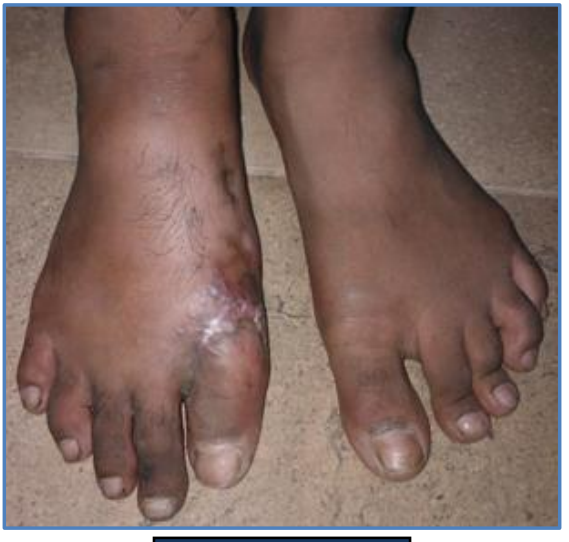

Figure 4b

Fig. 4a, b: a) immediate post-operative radiograph showing the k-wire in situ; b) 6 months postoperative clinical photograph.

DISCUSSION: Some conditions may be associated with this are- a) short, thick metatarsal; b) accessory toes; c) varus deformity of the other metatarsals; d) firm fibrous band on the medial side upto base of first metatarsal. The explanation behind origin of this deformity I that in utero two great toe originate, but the medial one gradually fails to develop. Later on the rudimentary toe along with the fibrous band acts as a taut bowstring and gradually pulls the fully developed great toe into a varus position.

There are few treatment options available for this deformity, and the choice should depend upon the severity of the deformity \&rigidity of the contracted soft tissue. For mild to moderate deformity Farmer's technique is appropriate, for severe deformity with excessive short first metatarsal operation of Kelikian is indicated. Arthrodesis may be needed in complicated cases in skeletally matured person ${ }^{3}$.

Tendon loop operation has also been described in papers using extensor hallusis longus in difficult cases 4 . We choose Farmer's procedure because the deformity was passively correctable and the metatarsal was not short, and the procedure was not much technically demanding.

\section{REFERENCES:}

1. Greig, DM. Hallux varus. Edinburg Medical journal, XXX, 588, 1923.

2. Farmer AW. Congenital hallux varus. American Journal of Surgery, 95:274, 1958

3. David Sloane. Congenital hallux varus-operative correction, JBJS vol- XVII, NO.1, JAN-1935.

4. S L Haas. An operation for the correction of Hallux varus, JBJS, VOL-XVI, 478, 1938. 


\section{CASE REPORT}

\section{AUTHORS:}

1. Arnab Karmakar

2. Tanmay Datta

3. Sunil Kumar Das

4. Ananda Kisore Pal

\section{PARTICULARS OF CONTRIBUTORS:}

1. Senior Resident, Department of Orthopaedics Surgery, IPGME\&R, Kolkata.

2. Assistant Professor, Department of Orthopaedics Surgery, IPGME\&R, Kolkata.

3. Associate Professor, Department of Orthopaedics Surgery, IPGME\&R, Kolkata.

4. Professor, Department of Orthopaedics Surgery, IPGME\&R, Kolkata.

\section{NAME ADDRESS EMAIL ID OF THE} CORRESPONDING AUTHOR:

Dr. Arnab Karmakar, Vil-Kushpata, Post-Ghatal, Dist-Paschim Midnapore, West Bengal - 721212.

E-mail: arnab.doctor@gmail.com

Date of Submission: 28/02/2014.

Date of Peer Review: 29/02/2014.

Date of Acceptance: 12/03/2014.

Date of Publishing: 02/04/2014. 